\section{Primary Androgen Deprivation Therapy for Nonmetastatic Prostate Cancer in Asia: Unique or Not?}

\author{
Masaki Shiota, MD, PhD
}

Sis ince the work of Huggins and Hodges in the mid 1900s, primary androgen deprivation therapy (PADT) has been the backbone therapy for metastatic prostate cancer. It has recently been combined with upfront docetaxel or abiraterone. Although PADT initially demonstrates excellent antitumor effects and palliates symptoms in most patients, castration-resistant disease often develops. Thus, PADT is recognized as being palliative rather than curative.

Although the survival benefit of PADT is vague, this treatment modality has been commonly used in clinical practice for nonmetastatic disease, especially in the elderly. However, 2 large cohort studies using a nationwide US database recently suggested that PADT has no long-term survival benefits in elderly men with localized prostate cancer. ${ }^{1,2}$ Subsequently, in the randomized EORTC 30891 trial, PADT showed a comparable outcome to observation (deferred ADT) in most cases of nonmetastatic disease, but not in very aggressive disease with a 3- to 5-year prognosis. ${ }^{3}$ Meanwhile, in the randomized SPCG-4 trial in Europe, therapy with radical prostatectomy (RP) showed a superior outcome to observation. ${ }^{4}$ Furthermore, the addition of radiotherapy to PADT for locally advanced disease was shown to improve survival compared with PADT alone in the randomized PR3/ PRO7 trial. ${ }^{5}$ Accordingly, it was suggested that radical therapies are likely to show superior survival to PADT or observation alone. Consequently, based on data obtained in Western countries, most guidelines, including the NCCN Clinical Practice Guidelines in Oncology (NCCN Guidelines) for Prostate Cancer, recommend active surveillance, radical therapy, or observation for nonmetastatic prostate cancer based on disease status and patient life expectancy. However, these guidelines recommend PADT as an option only for locally advanced disease if life expectancy is $\leq 5$ years and the patient is asymptomatic. ${ }^{6}$

Compared with white and black patients, PADT was suggested to be more effective in Asian populations. ${ }^{7}$ Furthermore, adverse effects associated with ADT, such as decreases in bone mineral density and possible increased risk of cardiovascular disease, are suggested to be milder in Asian versus Western populations. ${ }^{8}$ It was reported that the survival of patients with nonmetastatic prostate cancer was comparable to the life expectancy of the general population, ${ }^{9}$ although careful interpretation is required; men undergoing prostate biopsy, even for localized prostate cancer, are so-called healthy, whereas the general population includes very sick men. As a result, noninferior survival with PADT compared with radical therapy can be hypothesized as possible in Asian populations. Accordingly, in addition to the recommendation of PADT in the NCCN Guidelines, the NCCN Guidelines-Asia Consensus Statement for Prostate Cancer ${ }^{8}$ state that PADT may be considered as a possible treatment option for the following select patients: (1) low- and intermediate-risk patients with life expectancy $\geq 10$ years if they are unable to commit to radical therapy or active surveillance; (2) low- and intermediate-risk patients with life expectancy $<10$ years; and (3) high-risk patients, in whom ADT is suggested as a therapeutic option for those who are unfit or inaccessible for radical therapy, or have a short life expectancy.

However, is it true that PADT is an alternative therapy for radical therapy such as RP for nonmetastatic prostate cancer in Asian populations? In their article in this issue, $\mathrm{Ha}$ et $\mathrm{al}^{10}$ reported differential overall survival between RP and PADT using a health

See page 441 for related article.

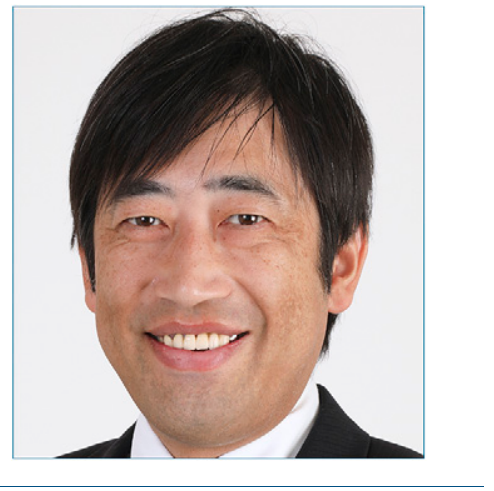

MASAKI SHIOTA, MD, PhD

Masaki Shiota, MD, PhD, is a Junior Associate Professor in the Department of Urology at Kyushu University, Fukuoka, Japan. Dr. Shiota has expertise in urological oncology, especially prostate cancer. $\mathrm{He}$ has worked on basic, translational, and clinical research in urological oncology. doi: $10.6004 /$ jnccn.2019.7302

The ideas and viewpoints expressed in this commentary are those of the author and do not necessarily represent any policy, position, or program of NCCN. 
insurance-based database linked with the cancer registry from South Korea. This study is not a randomized trial, and therefore could be biased with confounding factors. However, the biases were adjusted by propensity score matching (PSM) where possible, and thus provides a relatively robust answer to this question. The 5-year survival data clearly showed that overall survival is inferior for PADT compared with RP (5-year survival rate in PSM cohort, $77.7 \%$ vs $92.4 \%$, respectively) in nonmetastatic disease. This was consistently indicated in both localized and locally advanced disease. Thus, $\mathrm{Ha}$ et al refuted the hypothesis that PADT is a reasonable therapeutic option for nonmetastatic prostate cancer in Asian populations who are fit for and have access to radical therapy. Based on the findings obtained in this study, the recommendation in the NCCN Guidelines-Asia Consensus Statement to use PADT for low- and intermediate-risk patients with life expectancy $<10$ years was challenged.

This study has several limitations. First, the data on diagnosis, therapeutics, and outcome are very limited, and Gleason score, prostate-specific antigen level at diagnosis, detailed clinical stage and modality used for RP and PADT, and the events of progression and cancer-specific death are not available. Because the detailed data on diagnosis are missing, risk stratification and data analysis according to NCCN classification are impossible. The lack of detailed clinical stage in locally advanced disease is most critical, because it is one of the most important determinants of therapeutic strategy, wherein RP for T3aNO and radiotherapy for T3b/4NO, but PADT for TanyN1 would be preferred in many cases.

Second, this study is based on nonrandomized methods. In real-world settings, PADT is likely to be used in more advanced disease and for frailer patients compared with RP. Also, although the known biases were adjusted by PSM analysis, unknown biases could have affected the results. Third, other treatment modalities are lacking, such as radiotherapy, active surveillance, and observation. In particular, the outcomes of men who underwent radiotherapy would augment the results of this study, although this population is relatively small in South Korea.

Finally, one of the major limitations in this study is that the follow-up period is relatively short (median, $\sim 5$ years). Within this time-frame, the mortality event number is small, and in particular, cancer-specific death is estimated to be rare, although the data are not available. The inferior survival in PADT may be derived from non-cancer-specific death from causes other than cancer progression. Survival data are similar between localized and locally advanced disease, especially when treated with RP (5-year survival, $92.6 \%$ vs $76.5 \%$ with PADT, and $92.6 \%$ vs $71.1 \%$ with PADT, respectively). Interestingly, these data are almost identical to PADT survival data in EORTC 30891 (5-year overall survival, 70\%; 5-year cancer-specific survival, 90\%), ${ }^{3}$ suggesting a nonunique outcome of PADT in Asian patients.

Researchers have suggested that PADT may reduce cancer-specific death but increase non-cancer-specific death compared with observation, balancing the mortality rate between PADT and observation. ${ }^{3}$ Thus, men who are likely to die of metastatic prostate cancer may experience a beneficial outcome with PADT, whereas men unlikely to die of prostate cancer, such as those with nonmetastatic disease, may experience adverse effects resulting from PADT. Interestingly, the analysis of risk factors in overall survival revealed that osteoporosis was a risk factor of mortality, suggesting that the pathologic conditions associated with osteoporosis or induced by PADT might impair survival.

Thus, similar to the report from the United States, this study suggested that PADT also shows inferior outcomes compared with RP in Asian populations. Although careful judgement regarding the results is required due to the limitations, the study suggests important insights into the management of nonmetastatic prostate cancer in Asian men. An ongoing Japanese nationwide prospective cohort study by the Japan Study Group of Prostate Cancer, which is collecting data on the diagnosis, therapeutics, and longterm outcomes, is expected to contribute valuable information toward answering this question.

\section{Acknowledgments}

The author wishes to thank H. Nikki March, PhD, from Edanz Group (www.edanzediting.com/ac) for editing a draft of this manuscript.

\section{References}

1. Lu-Yao GL, Albertsen PC, Moore DF, et al. Fifteen-year survival outcomes following primary androgen-deprivation therapy for localized prostate cancer. JAMA Intern Med 2014;174:1460-1467.

2. Potosky AL, Haque R, Cassidy-Bushrow AE, et al. Effectiveness of primary androgen-deprivation therapy for clinically localized prostate cancer. J Clin Oncol 2014;32:1324-1330.

3. Studer UE, Whelan P, Wimpissinger F, et al. Differences in time to disease progression do not predict for cancer-specific survival in patients receiving immediate or deferred androgen-deprivation therapy for prostate cancer: final results of EORTC randomized trial 30891 with 12 years of follow-up. Eur Urol 2014;66:829-838.

4. Bill-Axelson A, Holmberg L, Garmo H, et al. Radical prostatectomy or watchful waiting in early prostate cancer. N Engl J Med 2014;370:932-942.

5. Warde $\mathrm{P}$, Mason M, Ding K, et al. Combined androgen deprivation therapy and radiation therapy for locally advanced prostate cancer: a randomised, phase 3 trial. Lancet 2011;378:2104-2111.

6. Mohler JL, Antonarakis ES, Armstrong AJ, et al. NCCN Clinical Practice Guidelines in Oncology: Prostate Cancer. Version 1.2019. Accessed March 12, 2019. To view the most recent version of these guidelines, visit NCCN.org.

7. Bernard B, Muralidhar $\mathrm{V}$, Chen $\mathrm{YH}$, et al. Impact of ethnicity on the outcome of men with metastatic, hormone-sensitive prostate cancer. Cancer 2017;123:1536-1544.

8. Akaza H, Umbas R, Ha Chung B, et al. NCCN Clinical Practice Guidelines in Oncology (NCCN Guidelines) - Asia Consensus Statement: Prostate Cancer. Version 2.2013. Available at NCCN.org. Accessed March 12, 2019.

9. Akaza H, Homma Y, Usami M, et al. Efficacy of primary hormone therapy for localized or locally advanced prostate cancer: results of a 10-year follow-up. BJU Int 2006;98:573-579.

10. Ha US, Choi JB, Shim JI, et al. Is primary androgen deprivation therapy a suitable option for Asian patients with prostate cancer compared with radical prostatectomy? J Natl Compr Canc Netw $2019 ; 17: 441-449$ 